ARTICLE

Received 11 May 2015 | Accepted 3 Sep 2015 | Published 9 Oct $2015 \quad$ DOl: 10.1038/ncomms9547 OPEN

\title{
Flexible, highly efficient all-polymer solar cells
}

\author{
Taesu Kim¹,2, Jae-Han Kim²,3, Tae Eui Kang ${ }^{1,2}$, Changyeon Lee ${ }^{1,2}$, Hyunbum Kang ${ }^{1,2}$, Minkwan Shin ${ }^{4}$, \\ Cheng Wang 5 , Biwu Ma ${ }^{6}$, Unyong Jeong ${ }^{4}$, Taek-Soo Kim²,3 \& Bumjoon J. Kim¹,2
}

\begin{abstract}
All-polymer solar cells have shown great potential as flexible and portable power generators. These devices should offer good mechanical endurance with high power-conversion efficiency for viability in commercial applications. In this work, we develop highly efficient and mechanically robust all-polymer solar cells that are based on the PBDTTTPD polymer donor and the $\mathrm{P}(\mathrm{NDI} / \mathrm{HD}-\mathrm{T})$ polymer acceptor. These systems exhibit high power-conversion efficiency of $6.64 \%$. Also, the proposed all-polymer solar cells have even better performance than the control polymer-fullerene devices with phenyl- $\mathrm{C}_{61}$-butyric acid methyl ester (PCBM) as the electron acceptor (6.12\%). More importantly, our all-polymer solar cells exhibit dramatically enhanced strength and flexibility compared with polymer/PCBM devices, with 60- and 470-fold improvements in elongation at break and toughness, respectively. The superior mechanical properties of all-polymer solar cells afford greater tolerance to severe deformations than conventional polymer-fullerene solar cells, making them much better candidates for applications in flexible and portable devices.
\end{abstract}

\footnotetext{
${ }^{1}$ Department of Chemical and Biomolecular Engineering, Korea Advanced Institute of Science and Technology (KAIST), Daejeon 305-701, Republic of Korea. 2 KI for the NanoCentury, KAIST, Daejeon 305-701, Republic of Korea. ${ }^{3}$ Department of Mechanical Engineering, KAIST, Daejeon 305-701, Republic of Korea.

${ }^{4}$ Department of Materials Science and Engineering, POSTECH, Pohang 790-784, Republic of Korea. ${ }^{5}$ Advanced Light Source, Lawrence Berkeley National Laboratory, Berkeley, California 94720, USA. ${ }^{6}$ Department of Chemical and Biomedical Engineering, Florida State University, Tallahassee, Florida 32310, USA. Correspondence and requests for materials should be addressed to T.-S.K. (email: tskim1@kaist.ac.kr) or to B.J.K. (email: bumjoonkim@kaist.ac.kr).
} 
T he emergence of flexible and wearable devices, such as electronic textiles, smart watches and patchable sensors, has stimulated research on alternative power generators for operating such devices. One of the basic requirements for these devices is that the integration of individual electronic components must retain flexibility and portability to function in new operational environments ${ }^{1-4}$. For this consideration, organic solar cells (OSCs) are currently viewed as promising power generation technologies that can be integrated into these devices because they are lightweight, semitransparent and flexible ${ }^{5,6}$. To date, most highly efficient OSCs have been based on polymerfullerene blends, in which fullerenes such as phenyl- $\mathrm{C}_{61}$-butyric acid methyl ester (PCBM) act as the electron acceptor. However, fullerenes are not ideal acceptor materials due to many intrinsic issues, such as weak light absorption and un-optimized energy levels, limiting the design adaptability of the electron donor pair. Moreover, fullerene-based OSCs have low flexibility and stretchability due to the brittle crystalline features of the fullerenes ${ }^{7,8}$. To resolve these drawbacks, a number of nonfullerene acceptors, including small molecules, nanoparticles and polymers, have been developed to replace fullerenes ${ }^{9-12}$.

All-polymer solar cells (all-PSCs), consisting of polymer-donor and polymer-acceptor materials, possess many advantages over polymer-fullerene solar cells, including tunable chemical and electronic properties as well as enhanced stabilities ${ }^{13-21}$. In recent years, many efforts have focused on developing optimal polymerdonor and polymer-acceptor combinations with well-controlled bulk-heterojunction $(\mathrm{BHJ})$ morphologies ${ }^{21-29}$. Among the nonfullerene acceptors, naphthalene diimide (NDI)-based copolymers have been the most successful polymer acceptors with high electron affinities and high electron mobilities, which are a result of their highly extended $\pi$-conjugated structure and strong $\pi-\pi$ intermolecular interaction ${ }^{30-35}$. The power-conversion efficiencies (PCEs) of all-PSCs have improved greatly to $5-6 \%$, and there is still great potential for further enhancement ${ }^{26-27,36-38}$, provided that simultaneous adjustment of polymer-donor and polymer-acceptor energy levels can improve their light harvesting and increase the open-circuit voltage $\left(V_{\mathrm{OC}}\right)$. Compared with conventional polymer-fullerene solar cells, all-PSCs can potentially exhibit much better mechanical strength and stability, because polymer acceptors are not only intrinsically more ductile than fullerenes but also are entangled with other polymers within the acceptor domain and at the interface ${ }^{39-41}$. In consideration of the application of PSCs in flexible devices, the mechanical properties of the all-PSCs should be investigated. However, to our knowledge, there is no report regarding this important topic.

Herein, we demonstrate that all-PSCs are better candidates than fullerene-based solar cells for applications in flexible and portable electronics. Highly efficient and mechanically robust
all-PSCs have been fabricated by using poly[4,8-bis(5-(2-ethylhexyl) thiophen-2-yl)benzo[1,2-b:4,5- $\left.\mathrm{b}^{\prime}\right]$ dithiophene-alt-1,3-bis(thiophen2-yl)-5-(2-hexyldecyl)-4H-thieno[3,4-c]pyrrole-4,6(5H)-dione] (PBDTTTPD) as the electron donor ${ }^{42-44}$ and poly[[ $N, N^{\prime}$-bis (2-hexyldecyl)-naphthalene-1,4,5,8-bis(dicarboximide)-2,6-diyl]-alt$5,5^{\prime}$-thiophene] (P(NDI2HD-T)) as the electron acceptor. The allPSCs based on this donor/acceptor pair of PBDTTTPD and $\mathrm{P}(\mathrm{NDI} 2 \mathrm{HD}-\mathrm{T})$ exhibited a PCE of 6.64\%, which is higher than that of PBDTTTPD:PCBM BHJ PSCs (PCE =6.12\%). The better performance of the all-PSCs was attributed to the high $V_{\mathrm{OC}}$ of $1.06 \mathrm{~V}$ and the optimized $\mathrm{BHJ}$ active layers of polymer donor and acceptor with favourable interfacial interactions. We have also studied the mechanical properties of the all-PSCs and found that, compared with fullerene-based blend films, all-polymer blend films offer superior flexibility, stretching and bending properties.

\section{Results}

Photovoltaic performances. Figure 1a shows the molecular structures and energy levels of PBDTTTPD, P(NDI2HD-T) and PCBM. The PBDTTTPD had number-average molecular weight $\left(M_{\mathrm{n}}\right)$ value of $22 \mathrm{~kg} \mathrm{~mol}^{-1}$ and optical bandgap of $2.02 \mathrm{eV}$ (see Supplementary Table 1), absorbing light in the wavelength of 400-650 nm (Fig. 1b). The P(NDI2HD-T) was also synthesized with $M_{\mathrm{n}}$ value of $48 \mathrm{~kg} \mathrm{~mol}^{-1}$, after considering that (1) the $\mathrm{P}(\mathrm{NDI} 2 \mathrm{HD}-\mathrm{T})$ had a higher lowest unoccupied molecular orbital energy level than that of PCBM, and (2) it had high electron mobility ${ }^{26}$. To explore the potential of these polymers in a photovoltaic system, we fabricated all-PSCs using a blend of PBDTTTPD and P(NDI2HD-T), and compared their performance with conventional polymer-fullerene solar cells (PCBMPSCs) based on a blend of PBDTTTPD and PCBM. Both PSCs were fabricated with the same device structure of ITO/poly-(3,4ethylenedioxythiophene):poly(styrenesulfonate) (PEDOT:PSS)/ blend layer/LiF/Al, although the processing conditions for the optimized PBDTTTPD:PCBM and PBDTTTPD:P(NDI2HD-T) blend layers were slightly different. For the PBDTTTPD:PCBM system, we used chloroform and 1,8-diiodooctane (DIO, 3 vol\%) as the processing solvents, and a polymer-donor concentration of $10 \mathrm{mg} \mathrm{ml}^{-1}$ with the donor:acceptor ratio of 1:1.5 (w/w). For the PBDTTTPD:P(NDI2HD-T) system, chloroform with $1 \mathrm{vol} \%$ of DIO was used to process a $12.5 \mathrm{mg} \mathrm{ml}^{-1}$ blend solution (1.3:1, w/w). The optimized film thicknesses of the PBDTTTPD:PCBM and PBDTTTPD:P(NDI2HD-T) blend layers were $\sim 200$ and $130 \mathrm{~nm}$, respectively. Details about the device optimization can be found in Supplementary Tables 2, 3 and 4. Figure 2 shows the current density versus voltage $(J-V)$ curves and external quantum efficiency (EQE) spectra of the optimized PSCs, and Table 1 summarizes the corresponding photovoltaic characteristics. The best PCE of the PCBM-PSCs was $6.12 \% \quad\left(V_{\mathrm{OC}}=0.96 \mathrm{~V}\right.$; a

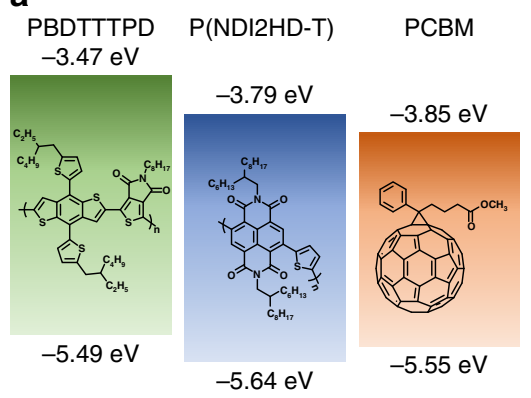

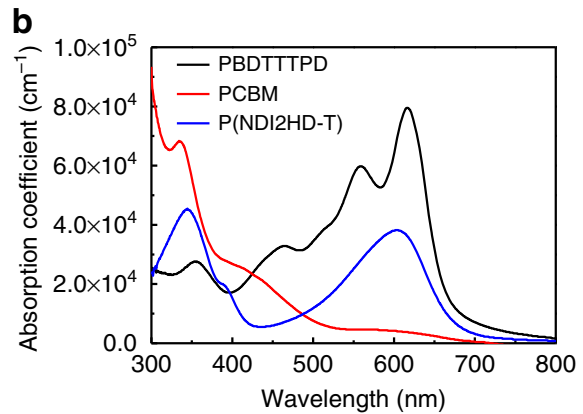

Figure 1 | Polymer information. (a) Chemical structures, energy levels, and (b) ultraviolet-visible absorption spectra of PBDTTTPD (black line), PCBM (red line) and $\mathrm{P}(\mathrm{NDI} 2 \mathrm{HD}-\mathrm{T})$ (blue line). 

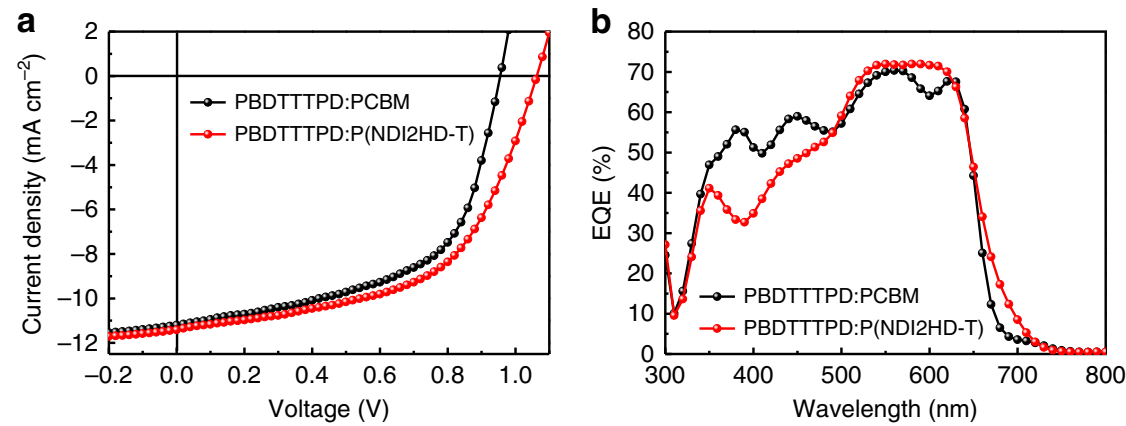

Figure 2 | J-V and EQE characteristics for the PCBM-PSC and the all-PSC. (a) J-V curves of normal-type devices, PBDTTTPD:PCBM (black line), PBDTTTPD:P(NDI2HD-T) (red line) under AM $1.5 \mathrm{G}$-simulated solar illumination (100 $\mathrm{mW} \mathrm{cm}^{-2}$ ); (b) EQE characteristics of the PBDTTTPD:PCBM (black line) and PBDTTTPD:P(NDI2HD-T) (red line).

\begin{tabular}{|c|c|c|c|c|c|c|c|c|}
\hline Device type & $V_{\text {oc }}(\mathrm{V})$ & $J_{s c}\left(m A c m^{-2}\right)$ & $\mathbf{F F}$ & $\begin{array}{c}\mathrm{PCE}_{\text {max }}{ }^{{ }^{\prime \prime}} \\
\left(\mathrm{PCE}_{\text {ave }}\right)^{+}(\%)\end{array}$ & $\begin{array}{c}J_{\mathrm{SC}}(E Q E) \ddagger \\
\left(\mathrm{mA} \mathrm{cm}^{-2}\right)\end{array}$ & $\left(\mathrm{cm}^{2} \mathbf{v}^{\mu_{\mathrm{h}}} \mathbf{1}^{-1}\right)$ & $\mu_{\mathrm{e}}\left(\mathrm{cm}^{2} \mathrm{v}^{-1} \mathrm{~s}^{-1}\right)$ & $\mu_{\mathrm{h}} / \mu_{\mathrm{e}}$ \\
\hline PCBM-PSCs & $0.96(0.959 \pm 0.003)$ & $11.17(11.208 \pm 0.057)$ & $0.57(0.565 \pm 0.007)$ & $6.12(6.076 \pm 0.045)$ & 10.89 & $2.52 \times 10^{-5}$ & $6.40 \times 10^{-5}$ & 0.4 \\
\hline All-PSCs & $1.06(1.062 \pm 0.001)$ & $11.22(11.243 \pm 0.028)$ & $0.56(0.553 \pm 0.006)$ & $6.64(6.601 \pm 0.058)$ & 10.96 & $2.84 \times 10^{-5}$ & $1.55 \times 10^{-5}$ & 1.8 \\
\hline
\end{tabular}

$J_{\mathrm{SC}}=11.17 \mathrm{~mA} \mathrm{~cm}^{-2}$; fill factor $\left.(\mathrm{FF})=0.57\right)$. This PCE value was consistent with or even higher than the PCE values of $4-6 \%$ reported in the literature for the PBDTTTPD:PCBM systems ${ }^{42-44}$. In contrast, when the $\mathrm{P}(\mathrm{NDI} 2 \mathrm{HD}-\mathrm{T})$ polymer was used as the electron acceptor, the best PCE increased significantly to $6.64 \%$ with $V_{\mathrm{OC}}$ of $1.06 \mathrm{~V}$, which represents one of the highest PCE and $V_{\mathrm{OC}}$ values reported to date for all-PSCs. The higher device performance of all-PSCs was mainly attributed to the enhanced $V_{\mathrm{OC}}$ value because of the higher-lying lowest unoccupied molecular orbital energy level of P(NDI2HD-T) than PCBM (Fig. 1a). We also measured the EQE spectra for the optimized PCBM-PSCs and all-PSCs. The $J_{\mathrm{SC}}$ values were well matched (within $3 \%$ error) with the integrated $J_{\mathrm{SC}}$ values obtained from the EQE spectra (Fig. 2b and Table 1). The EQE values of all-PSCs were higher than those of PCBM-PSC in the low-energy region of $500-700 \mathrm{~nm}$, but lower in the high-energy region of $350-500 \mathrm{~nm}$. This was not surprising if we considered the difference of the absorption for P(NDI2HD-T) and PCBM, that is, $\mathrm{P}$ (NDI2HD-T) having higher absorbance in the low-energy region but lower absorbance in the high-energy region compared with PCBM (Supplementary Fig. 1). Overall, comparable values of $J_{\mathrm{SC}}$ were obtained for both PSCs.

Polymer packing structure and blend morphology. To gain a deeper insight into the photovoltaic operation of the devices, we investigated the morphological characteristics of PBDTTTPD: PCBM and PBDTTTPD:P(NDI2HD-T) blends. First, we examined the polymer packing structures of PBDTTTPD:PCBM and PBDTTTPD:P(NDI2HD-T) blends via grazing incidence $\mathrm{X}$-ray scattering (GIXS) measurements (Supplementary Fig. 2). Supplementary Fig. 2a shows that the PBDTTTPD and $\mathrm{P}(\mathrm{NDI} 2 \mathrm{HD}-\mathrm{T})$ neat films had (100) scattering peaks in the inplane direction $\left(q_{\mathrm{xy}}\right)$ with lamellar domain spacings of $24.1 \AA$ $\left(q_{\text {in }}=0.26 \AA^{-1}\right)$ and $22.5 \AA\left(q_{\text {in }}=0.28 \AA^{-1}\right)$ and these scattering features were well preserved in the blend films (see Supplementary Fig. 2b). Prominent (010) peaks of PBDTTTPD:PCBM and PBDTTTPD:P(NDI2HD-T) blends, corresponding to $\pi-\pi$ stacking, were shown in the out-of-plane direction, indicating that both blends strongly preferred a face-on orientation relative to the substrate. The face-on stacked polymer blends should be beneficial for charge transport through the active layer to the electrodes ${ }^{18,45,46}$. Second, the blend morphologies of the PBDTTTPD:PCBM and PBDTTTPD:P(NDI2HD-T) films were characterized by atomic force microscopy (AFM) and resonant soft X-ray scattering (RSoXS) measurements (Supplementary Fig. 3). The AFM images showed that the PBDTTTPD:PCBM blend had coarser domains with a much larger surface roughness (root-mean-square value of $6.4 \mathrm{~nm}$ ) than that of the PBDTTTPD: $\mathrm{P}$ (NDI2HD-T) blend $(3.1 \mathrm{~nm})$. In addition, the RSoXS measurements showed that the scattering peak of the PBDTTTPD: $\mathrm{P}(\mathrm{NDI} 2 \mathrm{HD}-\mathrm{T})$ film had larger $q$ value $\left(0.0097 \AA^{-1}\right)$ with much weaker intensity compared with that of PBDTTTPD:PCBM blend film $\left(q=0.0036\right.$ and $\left.0.0083 \AA^{-1}\right)$, suggesting that PBDTTTPD: $\mathrm{P}(\mathrm{NDI} 2 \mathrm{HD}-\mathrm{T})$ blends possessed smaller domain size and much better intermixing ${ }^{26,47-49}$. The trend of RSoXS measurements was consistent with the results of the AFM measurements. The wellintermixed $\mathrm{BHJ}$ morphologies with smaller phase-separated domains for the PBDTTTPD:P(NDI2HD-T) blend could be partly attributed to the relatively low value of the interfacial tension $(\gamma)$ between the polymer donor and the polymer acceptor, which was estimated by the contact angle measurements (Supplementary Fig. 4 and Supplementary Table 5) ${ }^{50,51}$.

Space charge limited current charge mobility. We have also evaluated the hole mobility $\left(\mu_{\mathrm{h}}\right)$ and electron mobility $\left(\mu_{\mathrm{e}}\right)$ of the PBDTTTPD:PCBM and PBDTTTPD:P(NDI2HD-T) blends by using the space charge limited current measurements (Table 1 and Supplementary Fig. 5). The $\mu_{\mathrm{h}}$ values of the PCBM-based and 
the all-polymer-based blends were almost same, that is, $2.5 \times 10^{-5}$ and $2.8 \times 10^{-5} \mathrm{~cm}^{2} \mathrm{~V}^{-1} \mathrm{~s}^{-1}$, respectively. The $\mu_{\mathrm{h}}$ value of the PBDTTTPD:PCBM film was consistent with that reported previously $^{42}$. Both of the blends had $\mu_{\mathrm{e}}$ values with the same order of magnitude, that is, $10^{-5} \mathrm{~cm}^{2} \mathrm{~V}^{-1} \mathrm{~s}^{-1}$, with the $\mu_{\mathrm{e}}$ value of the PBDTTTPD:PCBM being higher. A $\mu_{\mathrm{h}} / \mu_{\mathrm{e}}$ value of 1.8 was obtained for the PBDTTTPD:P(NDI2HD-T) film, suggesting greatly more balanced hole and electron transports than those of other high-performance all-PSC systems ${ }^{17,21}$. Overall, the all-PSC system possessed desired features of polymer packing structure, blend morphology, and electrical properties, to achieve high performance, which were indeed better than or comparable to those of the fullerene-based PSC system.

Mechanical properties. The excellent mechanical stability of allPSCs represents another major merit for their potential applications in the portable and outdoor devices ${ }^{52-54}$. The sharp and weak interfaces between the polymer/fullerene junctions of the fullerene PSCs result in low cohesion and poor ductility in the $\mathrm{BHJ}$ active layer, and consequently mechanical fragility of the entire device ${ }^{7,54-57}$. In addition, the fullerenes in the blend film have strong tendency to crystallize, making the film even stiffer and more brittle with higher tensile moduli and lower cohesive energy with polymer donors ${ }^{8}$. Compared with PCBM-PSCs, allPSCs are expected to have much better mechanical properties owing to (i) the polymer acceptor's greater intrinsic flexibility than fullerenes, and (ii) the strengthened donor/acceptor interfaces by the entanglements between the polymer chains ${ }^{39,58}$.

To illustrate the difference of mechanical properties between PCBM-PSCs and all-PSCs, we first measured the tensile characteristics of PBDTTTPD:PCBM and PBDTTTPD: $\mathrm{P}$ (NDI2HD-T) blend films to obtain a quantitative comparison of the mechanical resilience of the two films (Fig. 3). We conducted a 'pseudo free-standing tensile test' (Supplementary Fig. 6), in which the PBDTTTPD:PCBM and PBDTTTPD: $\mathrm{P}(\mathrm{NDI} 2 \mathrm{HD}-\mathrm{T})$ thin films without any substrate were floated on the water surface without any significant damage to or wrinkling of the specimens ${ }^{56,59}$. Therefore, the intrinsic mechanical properties of the films, including tensile modulus and elongation at break, were directly measured without any substrate effects, complex calculations or assumptions. Figure 3a shows the stress-strain curves of the PBDTTTPD: PCBM and PBDTTTPD:P(NDI2HD-T) blend films. The elastic modulus and the elongation at break of the PBDTTTPD:PCBM $(1: 1.5 \mathrm{w} / \mathrm{w})$ blend film were measured to be $1.76 \mathrm{GPa}$ and $0.12 \%$, respectively. In addition to the optimized device condition of PBDTTTPD:PCBM $(1: 1.5 \mathrm{w} / \mathrm{w})$, we also performed a tensile test for the PBDTTTPD:PCBM blend with different blend ratio (1:0.5 $\mathrm{w} / \mathrm{w}$ ) and obtained a similar tensile modulus of $0.80 \mathrm{GPa}$ and an elongation at break of $0.30 \%$. The observed brittleness decreased as the PCBM content was lowered, which is consistent with what was previously reported and suggested that PCBM is the performance limiting component ${ }^{7,60}$. In contrast, and surprisingly, the tensile modulus of the PBDTTTPD:P(NDI2HD-T) blend film was only $0.43 \mathrm{GPa}$, and its elongation at break of PBDTTTPD:P(NDI2HD-T) blend was $7.16 \%$, which was a 60 fold enhancement over that of PBDTTTPD:PCBM. Note that considering the applications of all-PSCs in the wearable devices, this high value of elongation at break of our all-PSC satisfies the requirements ${ }^{61,62}$. These superior mechanical characteristics of all-PSC film than that of the PCBM-based BHJ film can provide a clear advantage for flexible and even stretchable electronic applications that require high tolerance against severe mechanical deformations. It should be noted that this is the first demonstration of all-PSC films with excellent mechanical resilience.

The excellent mechanical stability of the all-polymer films was also clearly confirmed by the calculation of toughness (Fig. 3b). The toughness of the BHJ films was obtained from the integration of the stress-strain curves in Fig. 3a. We found a remarkable contrast in the toughness values of the all-PSC films $\left(568.7 \mathrm{~J} \mathrm{~m}^{-3}\right)$ and the PCBM-PSC films $\left(1.2 \mathrm{~J} \mathrm{~m}^{-3}\right)$; the toughness of the all-PSC film was 470 times greater than that of the PCBM-PSC film. The tensile behaviours of the all-PSC film and PCBM-based film (1:0.5 w/w) were compared using an optical
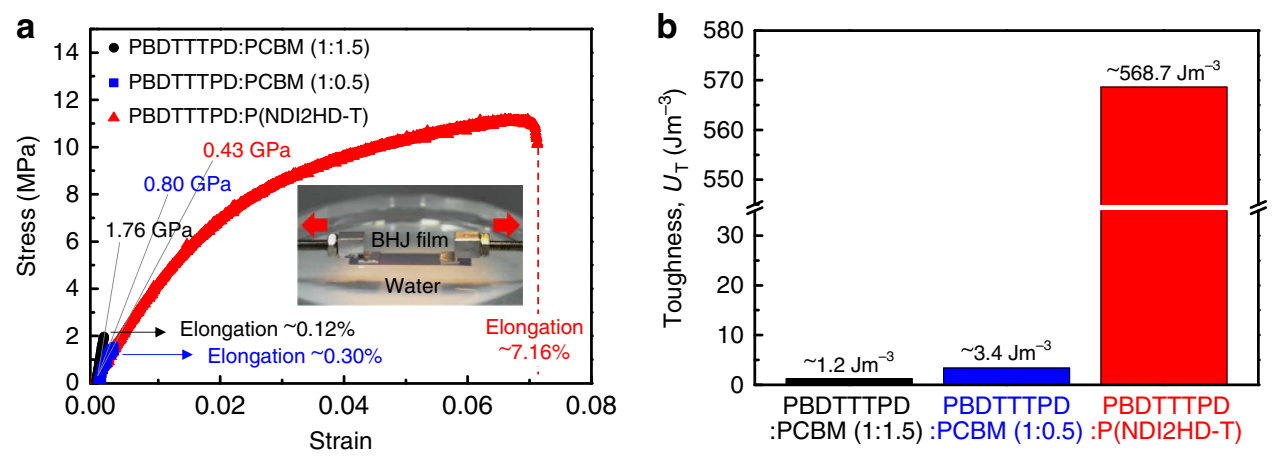

C

PBDTTTPD:PCBM

PBDTTTPD:P(NDI2HD-T)

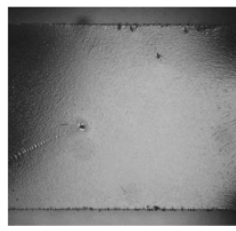

No elongation

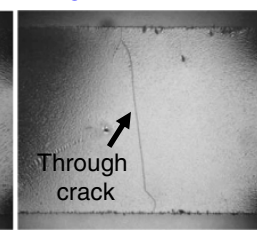

$0.3 \%$ elongation

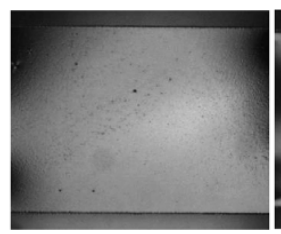

No elongation

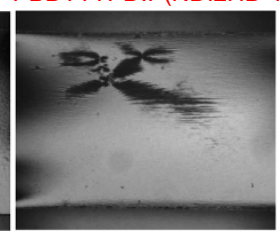

$6.3 \%$ elongation

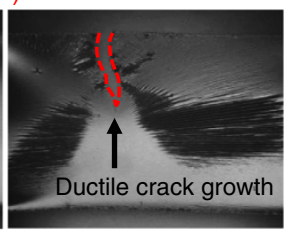

$7.0 \%$ elongation

Figure 3 | Tensile test of PBDTTTPD:PCBM and PBDTTTPD:P(NDI2HD-T) blend films. (a) Strain-stress curves and (b) toughness of PBDTTTPD:PCBM and PBDTTTPD:P(NDI2HD-T) blend films. (The inset in a shows photographs of the BHJ blend film floating on water. The specimens were gripped by the PDMS-coated Al grips and the films were prepared under the optimized device condition). (c) Optical microscopy images of PBDTTTPD:PCBM (1:0.5 w/w) and PBDTTTPD:P(NDI2HD-T) $(1.3: 1 \mathrm{w} / \mathrm{w})$ blend films when the films were under different strains. 
microscopy during the tensile tests (Fig. 3c). The results indicated that there was a dramatic difference in their fracture responses under tensile strain. The PCBM-based film had a sharp and through crack at very low value of elongation $(0.3 \%)$, representing its brittle nature. In contrast, the all-polymer film had a ductile nature, exhibiting no crack until high elongation of 7\%. This significantly improved mechanical property of the PBDTTTPD: $\mathrm{P}(\mathrm{NDI} 2 \mathrm{HD}-\mathrm{T})$ blend film can be attributed primarily to the ductility of the polymer films imposed by the entangled polymer chain $s^{39,40}$. In addition, the optimized $\mathrm{BHJ}$ morphologies of the PBDTTTPD:P(NDI2HD-T) blend with large interfacial area and well-intermixed polymer domains may contribute to the enhancement of their mechanical resilience ${ }^{63}$. The outstanding mechanical characteristics of the all-PSC films can enhance the ductility and the endurance against mechanical deformations by effectively relieving stress without mechanical failure, which is a critical requirement for flexible $\mathrm{PSC}^{52,53}$.

To further explore the potential of using all-PSCs in flexible devices, we measured the bending characteristics $3,64,65$ of the PBDTTTPD:PCBM and PBDTTTPD:P(NDI2HD-T) blend films. Their current-voltage $(I-V)$ characteristics were measured (Supplementary Fig. 7), and we obtained normalized conductance $\left[(\Delta I / \Delta V) /(\Delta I / \Delta V)_{0}\right]$ by monitoring the change in the $\Delta I / \Delta V$ values after bending to examine the stability of their electrical performances against external mechanical deformation (Fig. 4a). The $(\Delta I / \Delta V)_{0}$ is conductance value of the film before any bending. The blend films were prepared on a flexible polyimide substrate with a thickness of $80 \mu \mathrm{m}$. A 70-nm-thick $\mathrm{Au}$ electrode was thermally evaporated onto the films and the distance between the electrodes was $1 \mathrm{~mm}$. Figure $4 \mathrm{~b}$ compares the normalized conductance of the PBDTTTPD:PCBM and PBDTTTPD:P(NDI2HD-T) blend films after the bending test at different bending radii $(r=\infty, 3.0,1.9$ and $1.0 \mathrm{~mm})$. The electrical property of the fullerene-PSC film was reduced considerably at $r=1.0 \mathrm{~mm}$, compared with the reference sample at $r=\infty$. The apparent degradation was attributed to the crack propagation in the fullerene-PSC film by the mechanical deformation (Fig. 4d). In stark contrast, there was no change in the electrical and morphological properties of the all-PSC film, even at very small $r$ value of $1.0 \mathrm{~mm}$ (Fig. 4e). In addition, as shown in Fig. 4c, we compared the normalized conductance of the PBDTTTPD:PCBM and PBDTTTPD:P(NDI2HD-T) films after multiple cycles $(\mathrm{N}=0,50,100$ and 150) of bending at fixed $r=1.5 \mathrm{~mm}$. As $\mathrm{N}$ increased, the PBDTTTPD:PCBM blend film underwent a massive decrease of the current. However, the conductance of the all-PSC film was very stable, with only negligible changes at the same measurement conditions. The trend of the bending test fully corresponded to the results of the tensile modulus test, and the mechanical stability experiments consistently led to the same conclusion: the mechanical durability of all-PSCs is far superior to that of fullerene PSCs.

In summary, we have demonstrated highly efficient and mechanically robust all-PSCs. By using PBDTTTPD as the electron donor and P(NDI2HD-T) as the electron acceptor, allPSCs with high PCE of $6.64 \%$ have been achieved, which is even higher than that of control fullerene PSCs $(\mathrm{PCE}=6.12 \%)$. The enhanced performance of all-PSCs is mainly attributed to the high $V_{\mathrm{OC}}(1.06 \mathrm{~V})$ due to the better alignment of energy levels. Also playing significant roles are the enhanced absorption of $\mathrm{P}(\mathrm{NDI} 2 \mathrm{HD}-\mathrm{T})$ in the region of $500-700 \mathrm{~nm}$, as well as the desired a
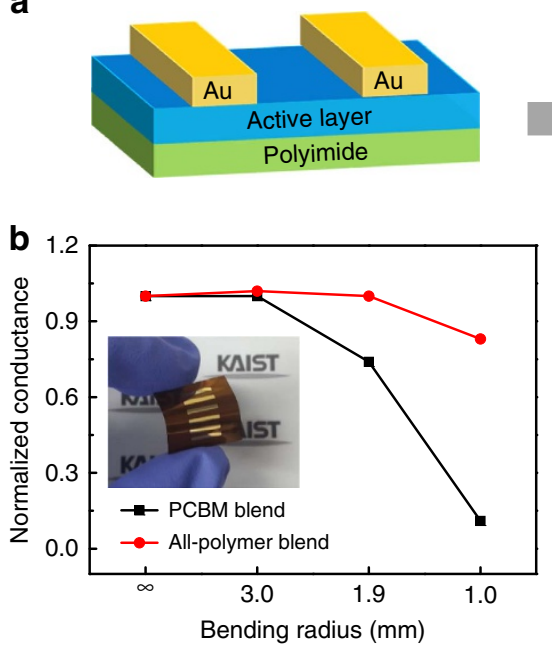

d

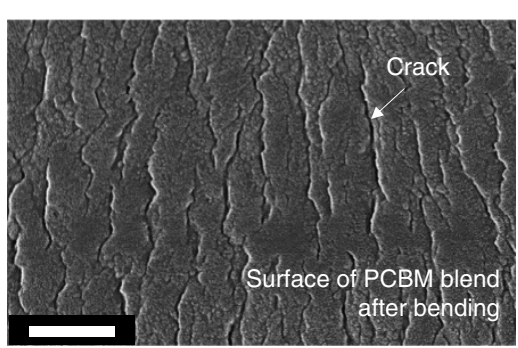

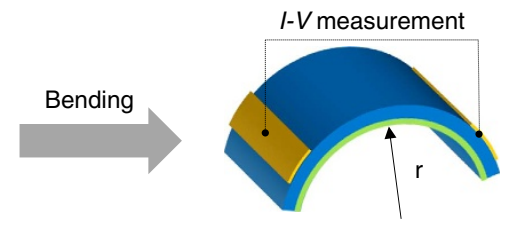
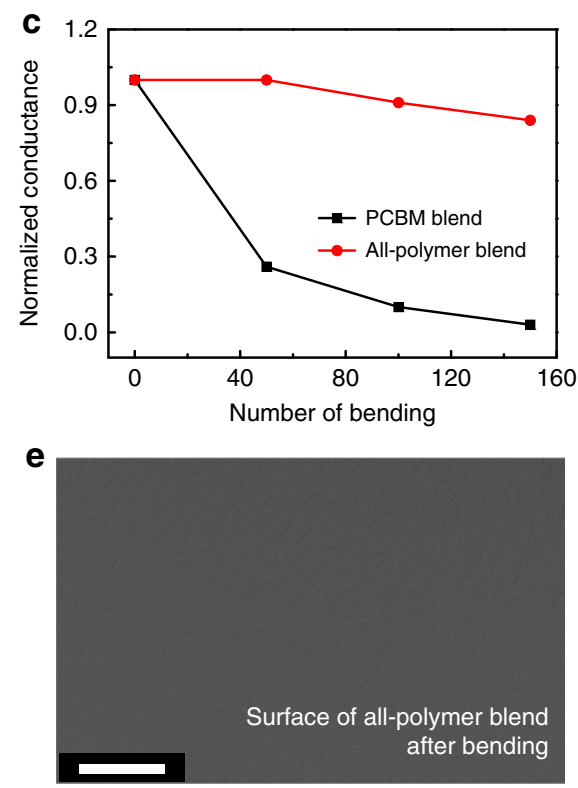

Figure 4 | Bending test of PBDTTTPD:PCBM and PBDTTTPD:P(NDI2HD-T) blend films. (a) Scheme of the BHJ blend film deposited on the flexible plastic substrate under mechanical bending. Normalized conductance of PBDTTTPD:PCBM and PBDTTTPD:P(NDI2HD-T) blend films (b) after bending at various $r$ values and (c) after multiple cycles of bending at $r=1.5 \mathrm{~mm}$. SEM images of surface morphologies of (d) PBDTTTPD:PCBM and

(e) PBDTTTPD:P(NDI2HD-T) blend films after bending at $r=1.0 \mathrm{~mm}$. The scale bars are $500 \mathrm{~nm}$. 
BHJ morphology of polymer blends with effective exciton dissociation and charge transport. More importantly, our study has shown that the all-PSCs have far superior mechanical durability compared with that of fullerene PSCs: the elongation at break and the toughness for all-PSCs are over 60 times and 470 times higher than those of the fullerene PSCs, respectively. This is due to the fact that the polymer acceptor is intrinsically more ductile than PCBM and can better entangle with other polymer chains with strengthened interfaces. And the superior electronic and mechanical performances of all-PSCs would make them more suitable for applications in flexible and portable devices than conventional polymer-fullerene PSCs. Our results provide guidelines for the design of new material systems for highperformance all-PSCs and demonstrate their potential for future applications in portable and wearable devices that require both high performances and mechanical stability.

\section{Methods}

Characterizations. Ultraviolet-visible absorption spectra were obtained using a UV-1800 spectrophotometer (Shimadzu Scientific Instruments) at room temperature. Static contact angles for water and glycerol were measured using contact angle analyzer (Pheonix 150, SEO, Inc.) equipped with a microsyringe that can dispense liquid droplets. AFM measurements were performed using a Veeco Dimension 3,100 instrument in tapping mode. The samples were prepared by spin coating onto PEDOT:PSS/ITO glasses. RSoXS measurements were performed at BL 11.0.1.2 in the Advanced Light Source (USA) using a series of photon energies to determine the maximum scattering contrast between the donor and the acceptors. RSoXS samples were prepared on a PEDOT:PSS/glass substrate under same optimized active layer condition. Then, the active layers were floated on water and transferred to a $1.0 \times 1.0 \mathrm{~mm}, 100$-nm-thick $\mathrm{Si}_{3} \mathrm{~N}_{4}$ membrane supported by a $5 \times 5 \mathrm{~mm}, 200-\mu \mathrm{m}$-thick Si frame (Norcada Inc.). GIXS measurements were performed at beamline $3 \mathrm{C}$ in the Pohang Accelerator Laboratory (South Korea). GIXS samples were prepared by spin coating onto a PEDOT:PSS/Si substrates. X-rays with a wavelength of $1.1179 \AA$ A were used. The incidence angle $\left(\sim 0.12^{\circ}\right)$ was chosen to allow for complete penetration of X-rays into the film.

\section{Device fabrication and measurement. The PCBM-PSCs and all-PSCs were} fabricated with an indium tin oxide (ITO)/PEDOT:PSS/(PBDTTTPD:PCBM (or PBDTTTPD:P(NDI2HD-T))/LiF/Al structure. ITO-coated glass substrates were subjected to ultrasonication in acetone, followed by extensive rinsing with deionized water and they were treated with ultrasonication in isopropyl alcohol. The substrates were then dried for several hours in an oven at $80^{\circ} \mathrm{C}$. The ITO substrates were treated with ultraviolet-ozone before PEDOT:PSS deposition. A filtered dispersion of PEDOT:PSS in water (PH 500) was applied by spin coating at 3,000 r.p.m. for $40 \mathrm{~s}$ and baking for $20 \mathrm{~min}$ at $150^{\circ} \mathrm{C}$ in air. After application of the PEDOT:PSS layer, all subsequent procedures were performed in a glove box under an $\mathrm{N}_{2}$ atmosphere. Then, each active blending solution was spin-cast onto an ITO/ PEDOT:PSS substrate at 1,000 r.p.m. for $60 \mathrm{~s}$ (or at 3,000 r.p.m. for 40 s). (Detailed preparation of the active layer solutions is described in the Supplementary Methods). The substrates were then placed in an evaporation chamber and held under high vacuum $\left(<10^{-6}\right.$ Torr) for more than $1 \mathrm{~h}$ before evaporating $\sim 0.9 \mathrm{~nm}$ of $\mathrm{LiF}$ and $100 \mathrm{~nm}$ of Al. The configuration of the shadow mask produced four independent devices on each substrate. The active area of the fabricated device was $0.09 \mathrm{~cm}^{2}$, which was carefully measured by optical microscope. The $J-V$ characteristics of the devices were measured under simulated AM 1.5G solar irradiation $\left(100 \mathrm{~mW} \mathrm{~cm}^{-2}\right.$,

Peccell: PEC-L01) at ambient condition. This solar simulator system satisfied the class AAB, ASTM standards. The intensity of the solar simulator was calibrated carefully by using a standard silicon reference cell with a KG-5 visible colour filter. The $J-V$ behavior was collected using a Keithley 2400 SMU. The EQE results were obtained using a spectral measurement system (K3100 IQX, McScience Inc.). This system applied monochromatic light from a xenon arc lamp at $300 \mathrm{~W}$ filtered by a monochromator (Newport) and an optical chopper (MC 2000 Thorlabs) at ambient conditions. The EQE data were obtained under dark conditions. The theoretical $J_{\mathrm{SC}}$ values were acquired by integrating the product of the EQE with the AM $1.5 \mathrm{G}$ solar spectrum and they were in good agreement with the measured $J_{\mathrm{SC}}$ to within $3 \%$ error.

Pseudo free-standing tensile test. For the tensile testing specimen, the active layers were spin-coated onto the PEDOT:PSS/glass substrate. The active layer specimen with a size of $2.54 \times 0.5 \mathrm{~cm}$ was prepared by using a cutting plotter (GCC Jaguar IV-61, USA). To float the specimen on the water surface, water was allowed to penetrate into the PEDOT:PSS layer. Subsequently, PEDOT:PSS was dissolved, and the active layer was delaminated from the glass substrate. By performing this process at the water surface, the floating active layer specimen could be obtained.
Specimen gripping was achieved by attaching PDMS-coated Al grips on the specimen gripping areas using van der Waals adhesion. The tensile test was performed by a linear stage with a strain rate of $0.06 \times 10^{-3} \mathrm{~s}^{-1}$. During the tensile test, stress and strain data were obtained through a load cell (LTS-10GA, KYOWA, Japan) and a digital image correlation (DIC) device, respectively.

Bending test. The $I-V$ curves were measured by a probe-station system (MST 8000C, HP 4156A).

\section{References}

1. Rogers, J. A., Someya, T. \& Huang, Y. Materials and mechanics for stretchable electronics. Science 327, 1603-1607 (2010).

2. Kim, D.-H. et al. Dissolvable films of silk fibroin for ultrathin conformal bio-integrated electronics. Nat. Mater. 9, 511-517 (2010).

3. Kaltenbrunner, M. et al. Ultrathin and lightweight organic solar cells with high flexibility. Nat. Commun. 3, 770 (2012).

4. White, M. S. et al. Ultrathin, highly flexible and stretchable PLEDs. Nat. Photon. 7, 811-816 (2013).

5. Thompson, B. C. \& Fréchet, J. M. J. Polymer-fullerene composite solar cells. Angew. Chem. Int. Ed. 47, 58-77 (2008).

6. Shrotriya, V. Organic photovoltaics: polymer power. Nat. Photon. 3, 447-449 (2009).

7. Lipomi, D. J., Chong, H., Vosgueritchian, M., Mei, J. \& Bao, Z. Toward mechanically robust and intrinsically stretchable organic solar cells: evolution of photovoltaic properties with tensile strain. Solar Energy Mater. Solar Cells 107, 355-365 (2012).

8. Savagatrup, S. et al. Mechanical degradation and stability of organic solar cells: molecular and microstructural determinants. Energy Environ. Sci. 8, 55-80 (2015).

9. Huynh, W. U., Dittmer, J. J. \& Alivisatos, A. P. Hybrid nanorod-polymer solar cells. Science 295, 2425-2427 (2002).

10. Anthony, J. E. Small-molecule, nonfullerene acceptors for polymer bulk heterojunction organic photovoltaics. Chem. Mater. 23, 583-590 (2011).

11. Zhou, E. et al. All-polymer solar cells from perylene diimide based copolymers: material design and phase separation control. Angew. Chem. Int. Ed. 50, 2799-2803 (2011).

12. Moore, J. R. et al. Polymer blend solar cells based on a high-mobility naphthalenediimide-based polymer acceptor: device physics, photophysics and morphology. Adv. Energy Mater. 1, 230-240 (2011).

13. Hwang, Y.-J., Ren, G., Murari, N. M. \& Jenekhe, S. A. n-type naphthalene diimide-biselenophene copolymer for all-polymer bulk heterojunction solar cells. Macromolecules 45, 9056-9062 (2012).

14. Facchetti, A. Polymer donor-polymer acceptor (all-polymer) solar cells. Mater Today 16, 123-132 (2013).

15. Zhou, E., Cong, J., Hashimoto, K. \& Tajima, K. Control of miscibility and aggregation via the material design and coating process for high-performance polymer blend solar Cells. Adv. Mater. 25, 6991-6996 (2013).

16. Mori, D., Benten, H., Okada, I., Ohkita, H. \& Ito, S. Highly efficient chargecarrier generation and collection in polymer/polymer blend solar cells with a power conversion efficiency of 5.7\%. Energy Environ. Sci. 7, 2939-2943 (2014)

17. Kang, H., Kim, K.-H., Choi, J., Lee, C. \& Kim, B. J. High-performance allpolymer solar cells based on face-on stacked polymer blends with low interfacial tension. ACS Macro Lett. 3, 1009-1014 (2014).

18. Schubert, M. et al. Correlated donor/acceptor crystal orientation controls photocurrent generation in all-polymer solar cells. Adv. Funct. Mater. 24, 4068-4081 (2014).

19. Zhou, Y. et al. High performance all-polymer solar cell via polymer side-chain engineering. Adv. Mater. 26, 3767-3772 (2014).

20. Mu, C. et al. High-efficiency all-polymer solar cells based on a pair of crystalline low-bandgap polymers. Adv. Mater. 26, 7224-7230 (2014).

21. Kang, H. et al. Determining the role of polymer molecular weight for highperformance all-polymer solar cells: its effect on polymer aggregation and phase separation. J. Am. Chem. Soc. 137, 2359-2365 (2015).

22. Kim, Y. et al. Organic photovoltaic devices based on blends of regioregular poly(3-hexylthiophene) and poly(9,9-dioctylfluorene-co-benzothiadiazole). Chem. Mater. 16, 4812-4818 (2004).

23. Li, Z. et al. Competitive absorption and inefficient exciton harvesting: lessons learned from bulk heterojunction organic photovoltaics utilizing the polymer acceptor P(NDI2OD-T2). Adv. Funct. Mater. 24, 6989-6998 (2014).

24. Li, W., Roelofs, W. S. C., Turbiez, M., Wienk, M. M. \& Janssen, R. A. J. Polymer solar cells with diketopyrrolopyrrole conjugated polymers as the electron donor and electron acceptor. Adv. Mater. 26, 3304-3309 (2014).

25. Jung, I. H. et al. Synthesis and search for design principles of new electron accepting polymers for all-polymer solar cells. Chem. Mater. 26, 3450-3459 (2014).

26. Lee, C. et al. High-performance all-polymer solar cells via side-chain engineering of the polymer acceptor: the importance of the polymer packing 
structure and the nanoscale blend morphology. Adv. Mater. 27, 2466-2471 (2015).

27. Hwang, Y.-J., Earmme, T., Courtright, B. A. E., Eberle, F. N. \& Jenekhe, S. A. $\mathrm{n}$-Type semiconducting naphthalene diimide-perylene diimide copolymers: controlling crystallinity, blend morphology, and compatibility toward high-performance all-polymer solar cells. J. Am. Chem. Soc. 137, 4424-4434 (2015).

28. Cheng, P. et al. Binary additives synergistically boost the efficiency of allpolymer solar cells up to 3.45\%. Energy Environ. Sci. 7, 1351-1356 (2014).

29. Mori, D., Benten, H., Okada, I., Ohkita, H. \& Ito, S. Low-bandgap donor/ acceptor polymer blend solar cells with efficiency exceeding 4\%. Adv. Energy Mater. 4, 1301006 (2014).

30. Steyrleuthner, R. et al. Bulk electron transport and charge injection in a high mobility n-type semiconducting polymer. Adv. Mater. 22, 2799-2803 (2010)

31. Kim, Y., Hong, J., Oh, J. H. \& Yang, C. Naphthalene diimide incorporated thiophene-free copolymers with acene and heteroacene units: comparison of geometric features and electron-donating strength of co-units. Chem. Mater. 25, 3251-3259 (2013).

32. Sommer, M. Conjugated polymers based on naphthalene diimide for organic electronics. J. Mater. Chem. C 2, 3088-3098 (2014).

33. Vasimalla, S., Senanayak, S. P., Sharma, M., Narayan, K. S. \& Iyer, P. K. Improved performance of solution-processed n-type organic field-effect transistors by regulating the intermolecular interactions and crystalline domains on macroscopic scale. Chem. Mater. 26, 4030-4037 (2014).

34. Yan, H. et al. A high-mobility electron-transporting polymer for printed transistors. Nature 457, 679-686 (2009).

35. Earmme, T., Hwang, Y.-J., Subramaniyan, S. \& Jenekhe, S. A. All-polymer bulk heterojuction solar cells with $4.8 \%$ efficiency achieved by solution processing from a co-solvent. Adv. Mater. 26, 6080-6085 (2014).

36. Jung, J. W. et al. Fluoro-substituted n-type conjugated polymers for additivefree all-polymer bulk heterojunction solar cells with high power conversion efficiency of 6.71\%. Adv. Mater. 27, 3310-3317 (2015).

37. Earmme, T., Hwang, Y.-J., Murari, N. M., Subramaniyan, S. \& Jenekhe, S. A. All-polymer solar cells with $3.3 \%$ efficiency based on naphthalene diimide-selenophene copolymer acceptor. J. Am. Chem. Soc. 135, 14960-14963 (2013).

38. Tan, Z.-K. et al. Suppressing recombination in polymer photovoltaic devices via energy-level cascades. Adv. Mater. 25, 4131-4138 (2013).

39. $\mathrm{Wu}, \mathrm{S}$. Chain entanglement and melt viscosity of compatible polymer blends: poly(methyl methacrylate) and poly(styrene-acrylonitrile). Polymer 28, 1144-1148 (1987).

40. Landel, R. F. \& Nielsen, L. E. Mechanical Properties of Polymers and Composites, Second Edition (Taylor \& Francis, 1993).

41. Jones, R. A. L. \& Richards, R. W. Polymers at Surfaces and Interfaces (Cambridge University Press, 1999).

42. Kang, T. E. et al. Importance of optimal composition in random terpolymerbased polymer solar cells. Macromolecules 46, 6806-6813 (2013).

43. Yuan, J. et al. Efficient polymer solar cells with a high open circuit voltage of 1 volt. Adv. Funct. Mater. 23, 885-892 (2013).

44. Warnan, J. et al. Ring substituents mediate the morphology of PBDTTPDPCBM bulk-heterojunction solar cells. Chem. Mater. 26, 2299-2306 (2014).

45. Piliego, C. et al. Synthetic control of structural order in N-Alkylthieno[3,4c]pyrrole-4,6-dione-based polymers for efficient solar cells. J. Am. Chem. Soc 132, 7595-7597 (2010).

46. Steyrleuthner, R. et al. The role of regioregularity, crystallinity, and chain orientation on electron transport in a high-mobility n-type copolymer. J. Am. Chem. Soc. 136, 4245-4256 (2014).

47. Collins, B. A. et al. Absolute measurement of domain composition and nanoscale size distribution explains performance in PTB7: $\mathrm{PC}_{71} \mathrm{BM}$ solar cells. Adv. Energy Mater. 3, 65-74 (2013).

48. Bartelt, J. A. et al. The importance of fullerene percolation in the mixed regions of polymer-fullerene bulk heterojunction solar cells. Adv. Energy Mater. 3 , 364-374 (2013).

49. Pavlopoulou, E. et al. Tuning the morphology of all-polymer opvs through altering polymer-solvent interactions. Chem. Mater. 26, 5020-5027 (2014).

50. Wu, S. Calculation of interfacial tension in polymer systems. J. Polym. Sci. Part C Polym. Symp. 34, 19-30 (1971).

51. Kim, K.-H. et al. Effects of solubilizing group modification in fullerene bis-adducts on normal and inverted type polymer solar cells. Chem. Mater. 24, 2373-2381 (2012).

52. Savagatrup, S., Printz, A. D., Rodriquez, D. \& Lipomi, D. J. Best of both worlds: conjugated polymers exhibiting good photovoltaic behavior and high tensile elasticity. Macromolecules 47, 1981-1992 (2014).
53. Savagatrup, S., Makaram, A. S., Burke, D. J. \& Lipomi, D. J. Mechanical properties of conjugated polymers and polymer-fullerene composites as a function of molecular structure. Adv. Funct. Mater. 24, 1169-1181 (2014).

54. Dupont, S. R., Voroshazi, E., Nordlund, D., Vandewal, K. \& Dauskardt, R. H Controlling interdiffusion, interfacial composition, and adhesion in polymer solar cells. Adv. Mater. Interfaces 1, 1400135 (2014).

55. Brand, V., Bruner, C. \& Dauskardt, R. H. Cohesion and device reliability in organic bulk heterojunction photovoltaic cells. Solar Energy Mater. Solar Cells 99, 182-189 (2012).

56. Kim, H. J. et al. Architectural engineering of rod-coil compatibilizers for producing mechanically and thermally stable polymer solar cells. ACS Nano $\mathbf{8}$, 10461-10470 (2014).

57. Bruner, C., Miller, N. C., McGehee, M. D. \& Dauskardt, R. H. Molecular intercalation and cohesion of organic bulk heterojunction photovoltaic devices. Adv. Funct. Mater. 23, 2863-2871 (2013).

58. Meijer, H. E. H. \& Govaert, L. E. Mechanical performance of polymer systems The relation between structure and properties. Prog. Polym. Sci. 30, 915-938 (2005).

59. Kim, J.-H. et al. Tensile testing of ultra-thin films on water surface. Nat. Commun. 4, 2520 (2013).

60. Awartani, O. et al. Correlating stiffness, ductility, and morphology of polymer:fullerene films for solar cell applications. Adv. Energy Mater. 3, 399-406 (2013).

61. Savagatrup, S., Printz, A. D., O’Connor, T. F., Zaretski, A. V. \& Lipomi, D. J. Molecularly stretchable electronics. Chem. Mater. 26, 3028-3041 (2014).

62. O'Connor, T. F. et al. Stretching and conformal bonding of organic solar cells to hemispherical surfaces. Energy Environ. Sci. 7, 370-378 (2014).

63. Harrats, C., Thomas, S. \& Groeninckx, G. Micro- and Nanostructured Multiphase Polymer Blend Systems: Phase Morphology and Interfaces (CRC Press, 2005).

64. Oh, J. Y. et al. Highly bendable large-area printed bulk heterojunction film prepared by the self-seeded growth of poly(3-hexylthiophene) nanofibrils. Macromolecules 46, 3534-3543 (2013).

65. Kim, M. et al. Lateral organic solar cells with self-assembled semiconductor nanowires. Adv. Energy Mater. 5, 1401317 (2014).

\section{Acknowledgements}

This research was supported by the National Research Foundation Grant (2013R1A2A1A03069803), and by the Global Frontier R\&D Program on Center for Multiscale Energy System (2012M3A6A7055540), funded by the Korean Government. Authors also acknowledge the KAIST-EEWS Research Project (EEWS-2012N01120012). We thank Professor Jung-Yong Lee and Gila Stein for helpful discussions

\section{Author contributions}

B.J.K., T.-S.K. and T.K. conceived of the experiments, analysed the data and wrote the manuscript. J.-H.K. measured the tensile properties. T.E.K. and C.L. synthesized the materials. H.K. and B.M. gave beneficial advices for polishing the experiments and the manuscript. M.S., T..K. and U.J. conducted the bending test. C.W. conducted the RSoXS measurements. T..K. prepared the samples and participated in all experiments. T.-S.K. and B.J.K. supervised the project. All authors discussed the results and contributed to the writing of the manuscript.

\section{Additional information}

Supplementary Information accompanies this paper at http://www.nature.com/ naturecommunications

Competing financial interests: The authors declare no competing financial interests

Reprints and permission information is available online at http://npg.nature.com/ reprintsandpermissions/

How to cite this article: Kim, T. et al. Flexible, highly efficient all-polymer solar cells. Nat. Commun. 6:8547 doi: 10.1038/ncomms9547 (2015).

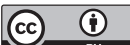

This work is licensed under a Creative Commons Attribution 4.0 International License. The images or other third party material in this article are included in the article's Creative Commons license, unless indicated otherwise in the credit line; if the material is not included under the Creative Commons license, users will need to obtain permission from the license holder to reproduce the material. To view a copy of this license, visit http://creativecommons.org/licenses/by/4.0/ 